\title{
Aprendizaje interactivo de termodinámica de fluidos apoyado en las tecnologías de la información y comunicación
}

\section{Interactive learning thermodynamics of fluids supported on information technologies and communication}

\author{
Edwin Rúa-Ramírez ${ }^{1}$, Andrea Barrera-Siabato ${ }^{2}$, Nidia Milena-Moreno-López ${ }^{3}$
}

Forma de citar: E. Rúa, A. Barrera, N. M. Moreno, "Aprendizaje interactivo de termodinámica de fluidos apoyado en las tecnologías de la información y comunicación”, Respuestas, vol. 19, no. 2, pp. 41-50, 2014.

Recibido:

Octubre 25 de 2013

Aceptado: Marzo 10 de 2014

\footnotetext{
${ }^{1}$ Magister en Termodinámica de Fluidos edwin.rua@unad.edu.co Universidad Nacional Abierta y a Distancia Yopal - Colombia
}

${ }^{2}$ Magister En Dirección y Administración De Empresas Universidad Nacional Abierta y a Distancia Yopal - Colombia

${ }^{3}$ Especialista en Educación y Orientación Familiar Universidad Nacional Abierta y a Distancia Tunja - Colombia

\section{Resumen}

Objetivo: El objetivo de este proyecto es diseñar un modelo de aprendizaje interactivo de la termodinámica de fluidos apoyado en las TIC e implementarlo a futuro en la Universidad Nacional Abierta y A Distancia UNAD y otras universidades. Métodos: Se realizó un diagnóstico del aprendizaje a través de una investigación descriptiva, de naturaleza cuantitativa y cualitativa con la aplicación de un cuestionario y análisis de datos a los 91 estudiantes matriculados en el II periodo 2012, en los CEAD de Yopal y Acacias, UNAD. Resultados: Los resultados del cuestionario fueron relevantes en el sentido de identificar factores críticos como el desconocimiento del software interactivo de termodinámica, falta de prácticas de laboratorio, ausencia de las TIC como apoyo a la docencia, falta de aulas virtuales y laboratorios para prácticas en las universidades. Conclusiones: Se pudo identificar algunos factores que influyen en el aprendizaje de la termodinámica, cual es el proceso actual de aprendizaje y algunos aspectos a mejorar. Se elaboró una metodología de enseñanza de la termodinámica apoyada en el software Termograf para implementar en los próximos semestres y evaluar su aplicación. Por fuentes bibliográficas se descubrió que el Termograf es una gran herramienta de aprendizaje autónomo que le permite al estudiante resolver diferentes problemas y al docente aplicar problemas reales con simulación y animación.

Palabras clave: Aprendizaje interactivo, Termodinámica de fluidos, Termograf, TIC.

\section{Abstract}

Objective: The objective of this Project is to design interactive learning as a model of thermodynamics of fluids, supported by ICT and implement 
it in the National Open University and Distance UNAD and other universities in future. Methods: A diagnosis of learning was conducted through a descriptive study of quantitative and qualitative nature by using a questionnaire as an inquiry tool and the corresponding analysis data; this was made with 91 students who were enrolled in the second period in 2012 at Yopal and Acacias CEADs, UNAD. Results: According to the inquiry results, it is important to say that they were relevant, because through them, some critical factors were identified, such as: The lack of interactive thermodynamics software, lack of ICT to support the teaching, lack of virtual classrooms and laboratories for achieving practices in universities. Conclusions: And also some factors related to the thermodynamics learning were identified, for instance, the current learning process and other issues about the thermodynamics, which should be improved. A teaching thermodynamics methodology was applied with the use of the thermograph software in order to be implemented for future semesters, so that, this application can be rehearsed and assessed.

Keywords: Learning interactive, Thermodynamics of fluids, Termograf, ICT.

\section{Introducción}

Tomando como punto de partida datos históricos universitarios que muestran el bajo rendimiento, cancelación y deserción de estudiantes en la asignatura Termodinámica, en el caso de la Universidad Nacional Abierta y a Distancia UNAD Zona AmazoníaOrinoquía, centros Yopal y Acacias, un alto porcentaje $(76 \%)$ de estudiantes matriculados en el curso 2012 II reprobó la asignatura, es evidente la dificultad que presentan frente al aprendizaje de este curso. Por tanto se hace necesario determinar las principales causas del bajo rendimiento en el aprendizaje. Se aplicó a través de un cuestionario virtual mediante "encuesta fácil" a los 91 estudiantes de termodinámica matriculados en la UNAD Zona AmazoníaOrinoquía con el fin de determinar hallazgos por el bajo rendimiento. La encuesta aplicada consta de 20 preguntas enmarcadas en los siguientes ítems, I. identificación de los factores que influyen en el aprendizaje de la termodinámica, II. Descripción del proceso actual del aprendizaje de la termodinámica,
III. Aspectos a mejorar en el proceso actual del aprendizaje de la termodinámica. El análisis de la encuesta reveló el desconocimiento de un software interactivo de termodinámica, falta de prácticas de laboratorio, metodología de enseñanza convencional docente - tablero, la no apropiación y uso de las TIC como apoyo a la docencia.

Con el fin de mejorar la metodología de enseñanza actual se busca aplicar un modelo de aprendizaje significativo de la termodinámica donde el estudiante relacione la información nueva con la de conocimientos previos que tiene, buscando aplicar las TIC como apoyo a través del software Termograf donde el estudiante interactúa desde cualquier computador con acceso a internet. Tomando esta hipótesis como posible solución se diseñan guías de laboratorio para realizar prácticas de forma virtual, se proponen y resuelven problemas de aplicación a la industria, se desarrollan problemas propuestos para analizar el comportamiento de las propiedades termodinámicas por medio de las tablas, los diagramas termodinámicos y 
gráficos que muestra el software. Obteniendo un aprendizaje practico, didáctico y autónomo saliendo de lo convencional, es decir de manera presencial alumno - profesor en un aula de clases.

Diferentes estudios han demostrado que los estudiantes tienen dificultades en la comprensión de los conceptos de calor y temperatura y tienen diferentes ideas a aquellas de las sostenidas por los científicos [1]. La complejidad en el entendimiento de las propiedades termodinámicas su comportamiento y relación, conllevan a buscar métodos o modelos de aprendizaje apoyados en las TIC que permitan obtener un aprendizaje más claro y real. El aprendizaje de la termodinámica de fluidos apoyado en las TIC es una opción muy viable e ideal para las nuevas y venideras generaciones cibernéticas como también para el modelo de educación virtual. [2]

\section{Materiales y métodos}

Se realizó una Investigación tipo descriptiva apoyada en un cuestionario - encuesta y se realizó el análisis de datos cuantitativos y cualitativos. Con base en la encuesta se pudo diagnosticar el estado actual de la enseñanza y aprendizaje de la Termodinámica de fluidos en la UNAD. Se diseñó una metodología de enseñanza interactiva de la termodinámica de fluidos apoyada en las TIC.

La población objetivo son los 91 estudiantes matriculados en la asignatura Termodinámica en el periodo 2012-2 de la Universidad Nacional Abierta y a Distancia UNAD Zona Amazonía-Orinoquía, centros Yopal y Acacias.

Instrumento: Encuesta enviada por e-mail y diseñada en el aplicativo Encuesta fácil.

http://www.encuestafacil. comRespWeb/ Cuestionarios.aspx?EID = 1480591 EMSJ = NO\#Inicio

Se diseñó un cuestionario estructurado en tres Ítems, con el fin de cumplir los tres objetivos del proyecto, este consta de veinte preguntas con tres escalas de respuesta. Los ítems fueron:

i. Identificación de los factores que influyen en el aprendizaje de la termodinámica de fluidos.

ii. Descripción del proceso actual del aprendizaje de la termodinámica de fluidos.

iii. Aspectos a mejorar en el proceso actual del aprendizaje de la termodinámica de fluidos.

Las preguntas y escalas de respuesta se muestran en las siguientes tablas:

Tabla I. ¿Cuál es el grado de importancia que usted considera de los siguientes factores que influyen en el aprendizaje de la termodinámica de fluidos?

\begin{tabular}{|c|c|c|c|c|c|c|}
\hline & & 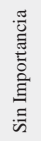 & 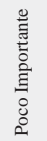 & 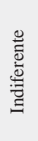 & 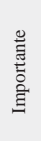 & 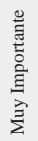 \\
\hline 1 & Variables Intrapersonales (Inteligencia o memoria) & & & & & \\
\hline 2 & Variables de personalidad (Actitud o fatiga) & & & & & \\
\hline 3 & Motivación intrínseca (Reconocimiento y estima) & & & & & \\
\hline 4 & $\begin{array}{l}\text { Motivación extrínseca (Retroalimentación Cognoscitiva, } \\
\text { estrategias de enseñanza) }\end{array}$ & & & & & \\
\hline 5 & Materiales de enseñanza (Libros, guías, manuales) & & & & & \\
\hline 6 & $\begin{array}{l}\text { Solución de problemas por cálculos realizados en } \\
\text { programas por computador. }\end{array}$ & & & & & \\
\hline 7 & Redes informáticas (Internet, Intranet) & & & & & \\
\hline 8 & Practicas interactivas por simulación en software & & & & & \\
\hline
\end{tabular}


No. 2

Julio-Diciembre 2014 ISSN 0122-820X

PP: 41-50

Tabla II. ¿Cuál es el estado actual de la metodología para el aprendizaje de la termodinámica de fluidos?

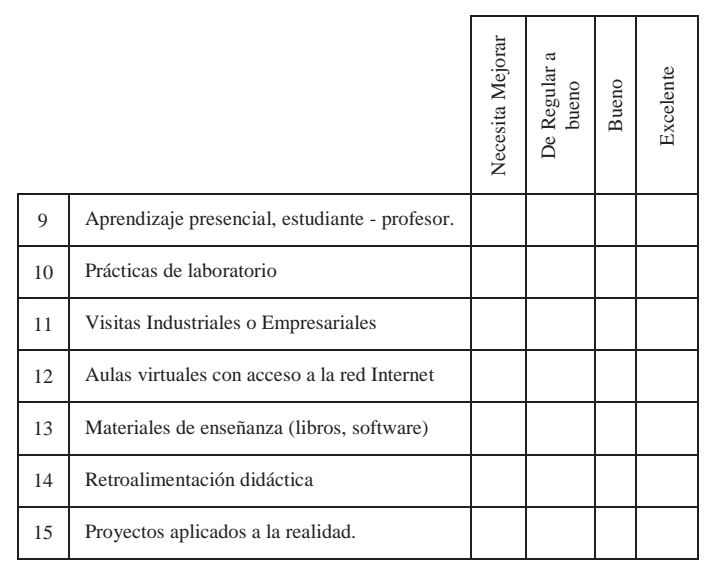

Fuente: Autores

Tabla III. ¿Cuál es el grado de usó en los siguientes aspectos necesarios para mejorar el proceso actual de aprendizaje de la asignatura termodinámica de fluidos?

\begin{tabular}{|c|c|c|c|c|}
\hline & & 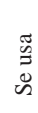 & $\begin{array}{l}\tilde{g} \\
0 \\
0 \\
\infty \\
0 \\
z\end{array}$ & $\begin{array}{ll} & 0 \\
\circ & \mathcal{O} \\
\circ & 0 \\
\mathrm{Z} & 0 \\
0\end{array}$ \\
\hline 16 & Uso de las Nuevas Tecnologías de información. & & & \\
\hline 17 & Resolución de problemas con simulación y animación. & & & \\
\hline 18 & $\begin{array}{l}\text { Análisis de diagramas, gráficas y tablas de propiedades } \\
\text { termodinámicas con programas didácticos. }\end{array}$ & & & \\
\hline 19 & $\begin{array}{l}\text { Representación visual de los conceptos con prácticas } \\
\text { interactivas. }\end{array}$ & & & \\
\hline 20 & $\begin{array}{l}\text { Elaboración de proyectos Industriales aplicados a los } \\
\text { problemas de la región. }\end{array}$ & & & \\
\hline
\end{tabular}

Fuente: Autores

Con este proyecto se pretende sustituir un aprendizaje memorístico basado en un débil seguimiento de la asignatura a lo largo del curso y un esfuerzo intenso pocos días antes del examen, por un trabajo sistemático e interactivo desarrollado desde el comienzo del curso, de forma que se posibilite no sólo la acumulación de conocimientos, sino el aprendizaje estructurado y significativo de los mismos. Se toma como objetivo el uso de las TIC para crear una metodología de aprendizaje interactivo y significativo. "Cuando el aprendizaje de los contenidos tiene lugar de forma significativa, lo que se posibilita es la autonomía del alumno para afrontar nuevas situaciones, para identificar problemas, para sugerir soluciones interesantes". [2]

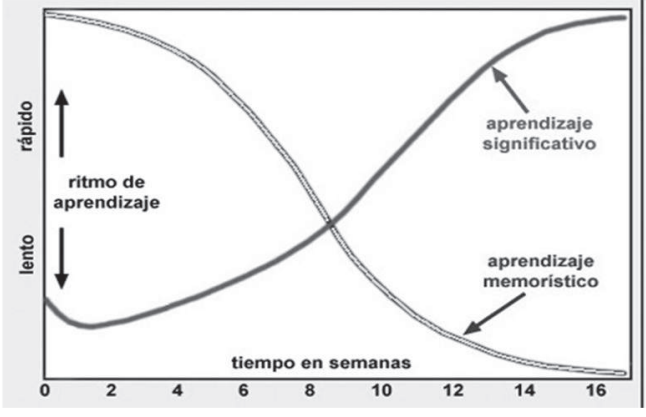

Figura 1. Evolución temporal del aprendizaje de conceptos según Novak.

Fuente: [3]

Por otra parte, se puede observar en la figura 1 la importancia que tiene en el proceso general de aprendizaje la forma de creación de conceptos que adopte un estudiante. Si adopta un aprendizaje de tipo memorístico, éste será eficaz en los primeros momentos, pero decaerá a largo plazo y desaparecerá al 
no ser capaz de resolver las interferencias con el nuevo aprendizaje que se produzca. Por el contrario, un aprendizaje significativo es inicialmente más lento, pero, a largo plazo, el recuerdo es más intenso y no hay interferencias sino refuerzo en el aprendizaje y en la estructuración de los conceptos. [3]

Con el diseño de esta metodología se plantea hacer uso de las TIC, para la obtención de resultados significativos en el aprendizaje, ya que esto implica la aplicación de varios principios, tales como: contar con objetivos claros, tener una buena estructuración de los materiales de aprendizaje, congruencia con las necesidades de los alumnos, entre otros, ofreciendo al alumno el trabajo individual además de distintas formas de abordar la información, mediante diversos estilos de aprendizaje, generando cambios y ventajas entre educación y nuevas tecnologías.

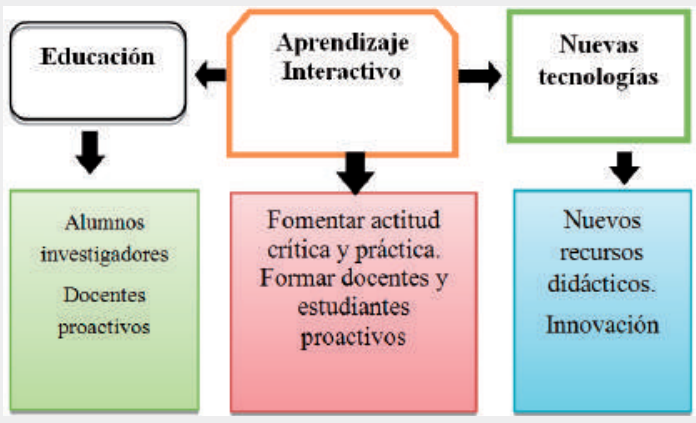

Figura 2. Cambios y ventajas de la articulación entre educación - nuevas tecnologías - transversalidad.

Fuente: Autores

\section{Resultados y análisis}

El análisis histórico de notas de la asignatura Universidad Nacional Abierta y A Distancia UNAD Zona Amazonía-Orinoquía, centros Yopal y Acacias, muestra de un total de 91 estudiantes matriculados en el segundo semestre del año 2012, de los cuales 66 reprobaron y 25 aprobaron el curso, como se muestra a continuación.

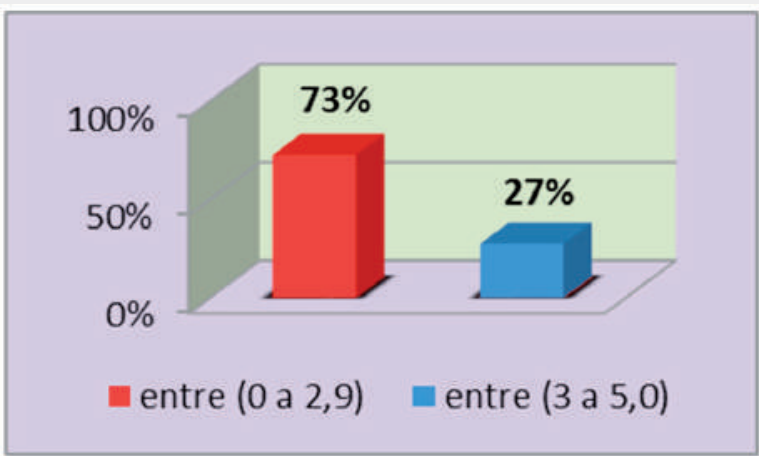

Figura 3. Estudiantes reprobados y aprobados en termodinámica año 2012, UNAD, zona zao.

Fuente: Autores

Los resultados del análisis de las encuestas arrojaron datos importantes, los estudiantes coincidieron en la ausencia de las TIC para el aprendizaje de la termodinámica, la falta de aulas virtuales con software especializado y laboratorios para el desarrollo de prácticas.

Julio-Diciembre 2014

ISSN 0122-820X 
Para el caso del material de enseñanza un 91,67 \% considera que los libros, guías y manuales son muy importantes.

Para el caso de las prácticas en software un 72,92 \% de los encuestados considera que es muy importante que se desarrolle prácticas interactivas por simulación en software

E1 93.3\% considera muy importante las estrategias de enseñanza que se implementan para mejorar el aprendizaje.

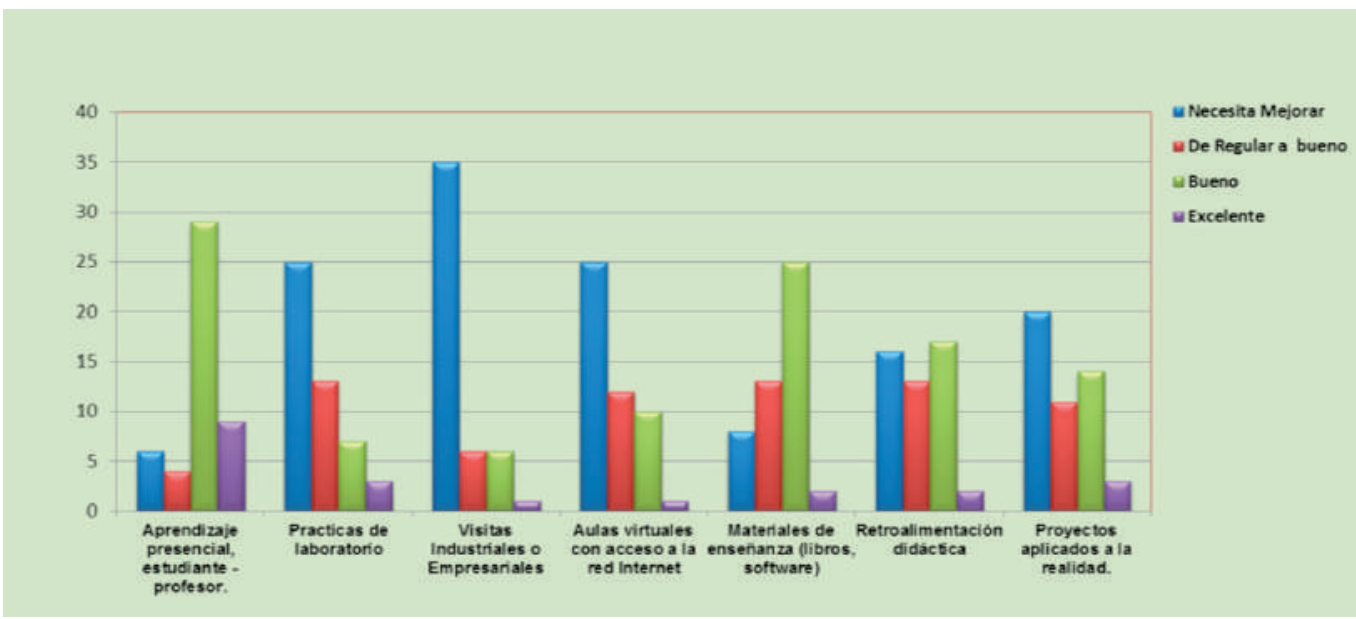

Figura 5. Estado actual de la metodología de enseñanza.

Fuente: Autores

Según los encuestados un 79,17 \% piensa que es necesario se mejore y aumente las prácticas de laboratorio.

Un 85,42 \% considera que es necesario se implementen y desarrollen visitas industriales con el fin de conocer de cerca equipos y procesos termodinámicos.

En el aspecto relacionado con las aulas virtuales un 77,08 \% considera que se deben mejorar en cuanto a acceso a la red y material disponible en la misma.

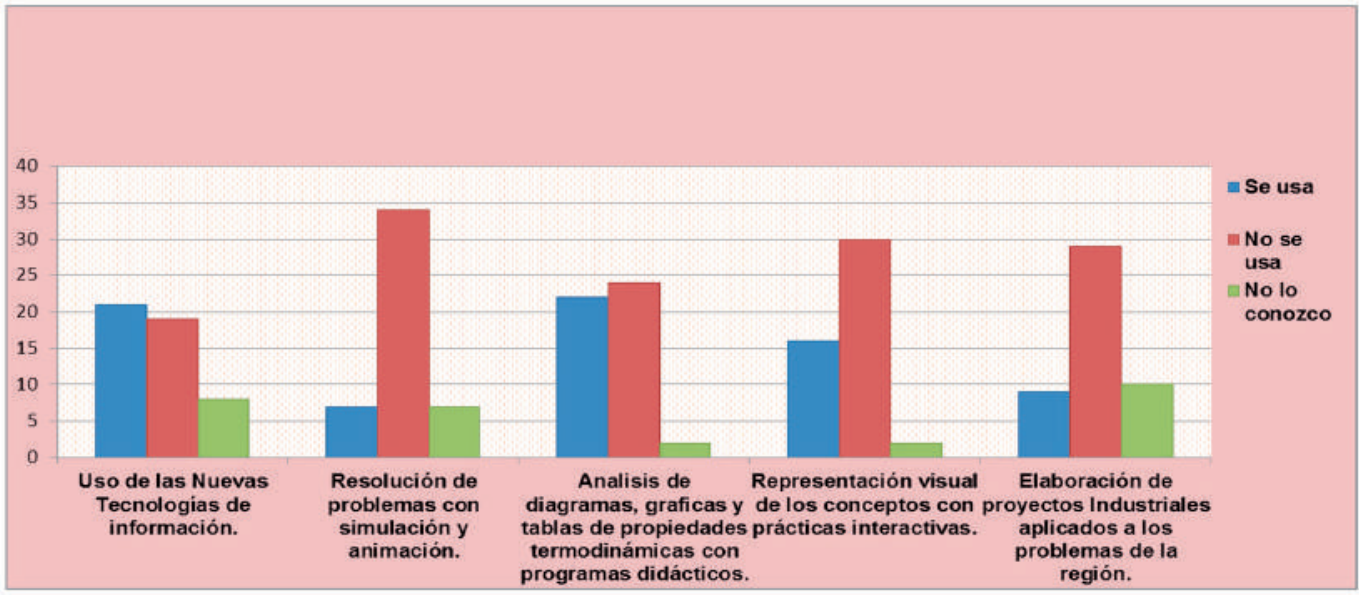

Figura 6. Uso de aspectos necesarios en la enseñanza de la termodinámica. 
El $85 \%$ de los encuestados asegura que no realizan simulación y animación para la resolución de problemas

El $72.5 \%$ no tienen practicas interactivas para la comprensión de los conceptos

El 71\% no elabora proyectos aplicados a los problemas industriales de la región

El 22.5\% dicen no conocer las nuevas tecnologías de la información como apoyo al aprendizaje interactivo.

El uso de las tecnologías para el aprendizaje autónomo e interactivo es beneficioso tanto para el estudiante como para el profesor. [4] Para la asignatura Termodinámica de fluidos existen programas para la determinación de las propiedades termodinámicas de algunas sustancias puras, también existe el software EES, (Engineering Equation Solver) para realizar cálculos de ingeniería y graficar, sin embargo el software termograf es un nuevo programa más completo y fue diseñado para la solución matemática, gráfica y simulada de problemas de termodinámica.

Con el diseño de esta metodología se busca mejorar la enseñanza y aprendizaje de la termodinámica en las Universidades de Colombia y así reducir el porcentaje de frustración de los estudiantes de ingeniería. Termograf, es una herramienta específica para la termodinámica que traslada el análisis de equipos e instalaciones energéticas a representaciones gráficas realistas, lo que facilita el trabajo requerido, pasando a un segundo plano la resolución de las complejas ecuaciones y centrando la atención del estudiante en la gestión de las magnitudes y conceptos relacionados con el problema/ ejercicio planteado.

Este software se considera una herramienta cognitiva en los términos que se describen en [5], una herramienta que garantiza un nivel de complejidad suficiente para incorporar una actividad real, yuxtaponiendo contenidos con acceso a distintos modos de representación, estimulando la percepción del propio pensamiento y enfatizando el protagonismo del estudiante [6].

La metodología planteada consiste en aplicar una enseñanza interactiva enfocada hacia un autoaprendizaje pedagógico y didáctico apoyado en el software TermoGraf, se maneja en la doble vertiente del entorno de enseñanza y del entorno de aprendizaje:

a) Entorno de enseñanza: El programa es utilizado en clase por parte del docente para explicar los conceptos de la asignatura de una forma práctica, utilizando de forma dinámica representaciones gráficas y cálculos con los distintos modelos y sustancias, pudiendo analizar con profundidad los diferentes procesos termodinámicos que tienen lugar en los sistemas e instalaciones térmicas [7].

b) Entorno de aprendizaje: Tiene fundamentalmente dos modos de uso, el uso de la sala informática para realización de prácticas informáticas y la resolución personalizada de ejercicios. Con ambos usos, se pretende que el estudiante trabaje de manera práctica los conceptos de la asignatura, tanto en clase orientados por el profesor/a, como en su vivienda (autoaprendizaje).

Termograf es una herramienta que abarca metódicamente todos los conceptos que se tratan a lo largo de la asignatura, por lo que es muy conveniente motivar a los estudiantes para que lo utilicen desde el principio, pues facilita altamente la comprensión de los conceptos implicados y sus interrelaciones, con una profundidad difícilmente alcanzable en el estudio convencional [8]. 


\section{Respuestas}

Cúcuta-Colombia

Vol. 19

No. 2

Julio-Diciembre 2014

ISSN 0122-820X

PP: 41-50
Aprendizaje interactivo de termodinámica de fluidos apoyado en las tecnologías de la información y comunicación

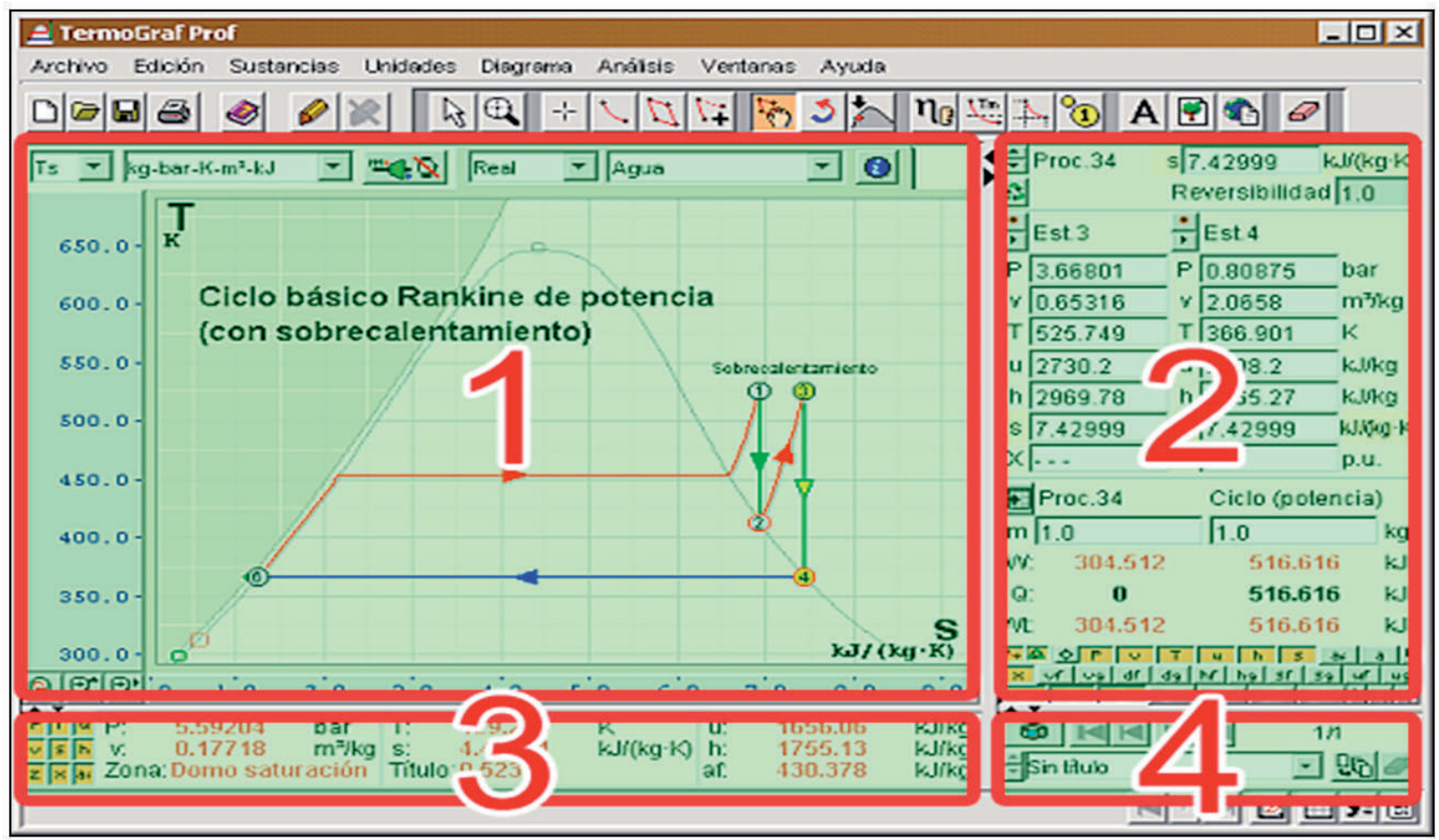

Figura 7. Ventana principal de Termograf, versión profesor.

Fuente: [9].

La versión de profesor es la que permite la edición, corrección y evaluación de las pruebas planteadas a los alumnos, como muestra la figura 3, para esta forma de empleo, Termograf posee un modo de trabajo específico, el modo ejercicio, en el cual el estudiante obtiene una pantalla previamente preparada por el profesor/a, con los enunciados de los ejercicios a realizar [10].

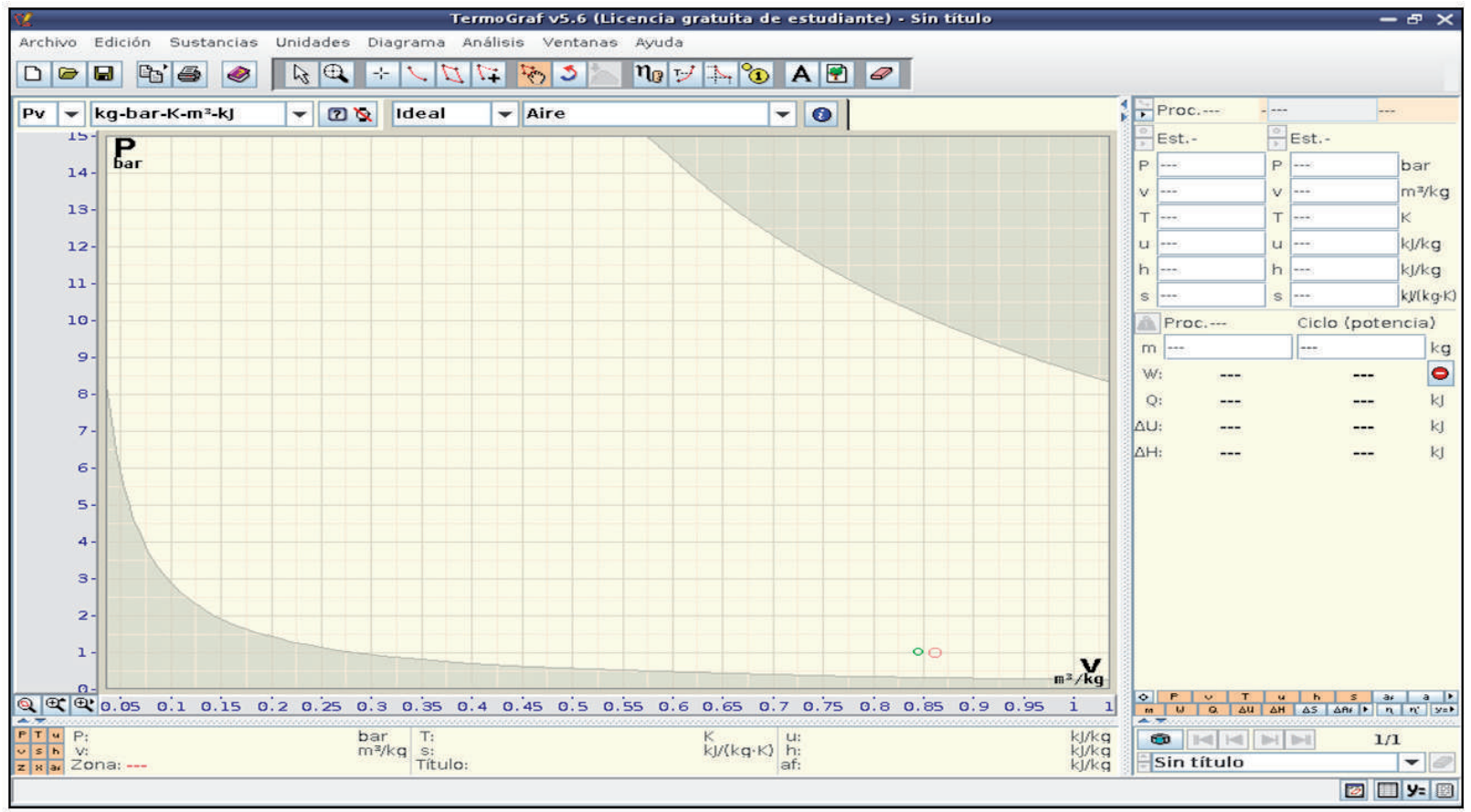

Figura 8. Termograf versión estudiante. Fuente: [11]. 
La versión gratuita para el estudiante es una herramienta de apariencia muy simple que contiene: Visualización del enunciado del ejercicio, con un panel adjunto para introducir las respuestas. Editor de ecuaciones y creación de variables personales para la resolución de sistemas de ecuaciones.

Uno de los principales retos en el uso de las TIC como apoyo a la educación, es superar la resistencia al cambio por parte de los profesores de educación convencional, pero no se trata de refutar con las nuevas tecnologías antiguos programas educativos, sino de diseñar y aplicar modelos pedagógicos de innovación que orienten a los alumnos hacia las competencias que necesitarán para adaptarse en un mundo que ya es digital.

La investigación realizada en la UNAD, en el curso de Termodinámica 2012-2, indicó que el $73 \%$ de los estudiantes reprobaron la asignatura por el método de aprendizaje convencional, a partir de este estudio se pretende aplicar la nueva metodología de enseñanza apoyada en las TIC donde el docente se debe involucrar en el manejo del software termograf con el apoyo de los tutoriales, animaciones, simulaciones y proponer unos ejercicios para aplicar los conceptos de la asignatura de una forma práctica.

Para la aplicación de la metodología creada se necesita una sala de informática y a través de la orientación del profesor el estudiante aprende los conceptos con mayor destreza. A partir de esta investigación se proyecta aplicar el próximo semestre esta metodología a los estudiantes de la UNAD y comparar con los estudios previos, en caso de mejorar el rendimiento académico se demostraría a la comunidad universitaria de Colombia que el aprendizaje apoyado en las TIC produce resultados de aprendizaje estructurados, lo cual facilita que permanezcan a lo largo del tiempo de forma más sólida que en el memorístico.

\section{Conclusiones}

En esta investigación se pudo diagnosticar que la pedagogía convencional no es la apropiada para el curso virtual de Termodinámica de la UNAD ya que es muy teórica y los estudiantes no logran comprender los conceptos, la relación y aplicación de tantas propiedades termodinámicas en cada estado, proceso o ciclo, les dificulta asimilar los diferentes diagramas y los cambios de fase de cada sustancia.

Se pudo identificar que algunos factores influyen en el aprendizaje de la termodinámica y existen algunos aspectos a mejorar en el proceso actual de aprendizaje. Se confirmó que el modelo de enseñanza actual es teórico, no existen prácticas de laboratorio ni visitas industriales.

El $73 \%$ de los estudiantes matriculados en termodinámica durante 2012-2 de Universidad Nacional Abierta y A Distancia UNAD Zona Amazonía-Orinoquía, centros Yopal y Acacias, perdieron la asignatura.

Este proyecto busca aplicar una nueva metodología de enseñanza en el curso de Termodinámica de la UNAD, que integre los conceptos básicos teóricos de la termodinámica con aplicaciones prácticas desarrolladas en un software que le permita al estudiante aprender a través de gráficas, animación y simulación problemas reales y procesos industriales.

Un 79,17 \% de los estudiantes encuestados piensa que es necesario que se mejore y aumente las prácticas de laboratorio en termodinámica, Un 85,42 \% considera que es necesario se implementen y desarrollen visitas industriales con el fin de conocer de cerca equipos y procesos termodinámicos y un 77,08 $\%$ considera que se debe mejorar o cambiar la estrategia de enseñanza - aprendizaje convencional.

El curso actual de termodinámica de la UNAD emplea la estrategia de aprendizaje basado en problemas $(\mathrm{ABP})$ con ejercicios de aplicación a la Industria, sin embargo el estudiante no
Julio-Diciembre 2014

ISSN 0122-820X

PP: $41-50$ 
No. 2

Julio-Diciembre 2014 ISSN 0122-820X

PP: 41-50 percibe claramente el comportamiento de los fluidos en un proceso o ciclo termodinámico. Con la nueva estrategia de aprendizaje interactivo apoyado en las TIC, se busca que el estudiante interactúe constantemente con el Termograf, ya que esta herramienta le va facilitar la comprensión de los conceptos, le va ahorrar tiempo en el desarrollo de los problemas y le va permitir observar el comportamiento de los fluidos y relación de las propiedades termodinámicas en un estado, proceso o ciclo.

\section{Referencias}

[1] A. Cervantes, "Los conceptos de calor y temperatura: una revisión bibliográfica”, Enseñanza de las Ciencias, vol. 5, no. 1, pp. 66-70, 1987.

[2] C. Coll y I. Solé. "Aprendizaje significativo y ayuda pedagógica", Revista Candidus, vol. 15, pp. 19-27, 2001.

[3] M. C. Velasco, B. Zalba, J. C. Rafales, M. A. Hernández. "Un entorno de enseñanza-aprendizaje apoyado en las tic para coordinar teoría, prácticas de simulación y trabajo personal en cursos de ingeniería térmica y termodinámica aplicada" X Congreso de Innovación Educativa en las Enseñanzas Técnicas. Valencia, 2002.

[4] F. Barriga, G. Hernández, Estrategias docentes para un aprendizaje significativo, una interpretación constructivista. 2da edición. México: Mc Graw Hill, 2012.

[5] J. A. Turegano, C. Velazco "Resultados de la incorporación de la evaluación continua como integración de los entornos de enseñanza y aprendizaje apoyados en las tic" Universidad de Zaragoza, Grupo de didáctica en energía térmica, 2012.

[6] J. M. Martínez, B. A. Pérez, "Estudio de

[8] U. Zaragoza, Grupo didáctica en ingeniería térmica. Termograf simulador termodinámico, [Online]. Disponible en: http://termograf.unizar.es/www/ caracteristicas/termodinamica.htm

[9] U. Zaragoza, Grupo didáctica en ingeniería térmica. Termograf simulador termodinámico, Docencia de la termodinámica, [Online]. Disponible en: http://termograf.unizar. es/www/caracteristicas/educacion.htm

[10] T. Gómez, M. C. Velasco, "Evaluación continua en Ingeniería Térmica y difusión internacional del mismo mediante una red colaborativa de profesores”, Zaragoza Julio 2010.

[11] U. Zaragoza, Grupo didáctica en ingeniería térmica Termograf simulador termodinámico, Docencia de la termodinámica, [Online]. Disponible en: http://termograf.unizar.es/www/ docum/tutoriales.htm

propuestas alternativas en la enseñanza de la termodinámica básica, Enseñanza de las ciencias" Universidad de la Patagonia, Argentina, pp. 287 - 300,

[7] R. Oñate García, I. Sánchez Soto, "Resolución de problemas por investigación y su influencia en los Trabajos Prácticos de Laboratorio en Termodinámica", Revista de Pedagogía, vol. 31, no. 89, pp. 307-329, JulioDiciembre de 2010.

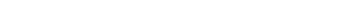

